\title{
REVISIÓN DE LA BEST PRACTICE “RED ESPAÑOLA DE CIUDADES POR EL CLIMA", DESDE LA ESTRATEGIA EUROPEA MEDIOAMBIENTAL
}

Rafael Córdoba Hernández y Agustín Hernández-Aja²

\section{Contexto}

En el siglo pasado la comunidad internacional comenzó un período de reflexión relacionado con los problemas ambientales de nuestro planeta, que ha llegado hasta hoy, situándose en el centro de discusión el problema del cambio climático.

El modelo territorial impuesto ha contribuido notablemente al aumento del problema, teniendo como principal exponente el modelo de ordenación territorial y urbana. Gran parte de la transformación

1 España. Arquitecto urbanista Escuela Técnica Superior de Arquitectura de Madrid, Universidad Politécnica de Madrid. PROES Consultores. Email: rafaelcordobahernandez@gmail.com

2 España. Doctor arquitecto. Director del Departamento de Urbanística y Ordenación del Territorio y miembro del GIAU+S de la Escuela Técnica Superior de Arquitectura de la Universidad Politécnica de Madrid Email: agustin.hernandez@upm.es está en poder de los municipios, que tienen la responsabilidad de modificar el diseño y gestión de las áreas urbanas, así como del desarrollo y ejecución de políticas públicas para enfrentarnos a estos desafíos.

Los programas presentados por los gobiernos nacionales o las agencias de la UE, pueden parecer ajenos o de difícil gestión por los municipios, pero en realidad sus atribuciones son adecuadas para atender a las realidades territoriales y además, de mayor comprensión por los ciudadanos. 


\section{PROGRAMA DE ACCIÓN EN MATERIA DE MEDIO AMBIENTE}

Una de las principales políticas medioambientales europeas, en relación al cambio climático, es el VI Programa de Acción en Materia de Medio Ambiente de la Unión Europea 2002-2012. Su principal meta en este ámbito es ayudar a "estabilizar las concentraciones en la atmósfera de gases de efecto invernadero en un nivel que impida la interferencia antropogénica peligrosa en el sistema climático"3. Para ello elabora una serie de objetivos:

1. Compromisos climáticos internacionales.

2. Reducir emisiones en sector energético.

3. Reducir emisiones en sector transportes.

4. Reducir emisiones en producción industrial.

5. Reducir emisiones en otros sectores.

6. Usar otros instrumentos adecuados.

\section{LA RED ESPAÑOLA DE CIUDADES POR EL CLIMA}

Los municipios españoles, agrupados en la Federación Española de Municipios y Provincias ${ }^{4}$, crean en 2005 la Red Española de Ciudades por el Clima, para desarrollar una metodología con un sistema de indicadores para estimar las emisiones de gases de efecto invernadero producidos en los municipios, con el objetivo de que sea común, para desarrollar estudios comparativos de manera objetiva.

La red tiene una dinámica propia y un interés evidente, al reflejar la voluntad de los municipios que participan en ella, de actuar con el objetivo de reducir el impacto de las ciudades sobre el medio ambiente global. Aunque puede existir un gran número de iniciativas municipales que no encuentren su reflejo en la red, se entiende que ésta garantiza un criterio de calidad e interés suficiente para que merezcan ser estudiadas.
3 UNIÓN EUROPEA (2002). VI Programa de Acción Comunitaria en materia de Medio Ambiente para el periodo 2002-2012. Diario Oficial de las Comunidades Europeas. L 242. Artículo 2. Principios y metas generales
4 También denominada FEMP. Es una asociación española de entidades locales que agrupa ayuntamientos, diputaciones provinciales y la diputación foral de Álava, consells insulars y cabildos insulares. Quedó constituida en la Disposición Adicional Quinta de la Ley 7/1985, de 2 de abril, Reguladora de las Bases de Régimen Local y fue declarada como Asociación de Utilidad Pública mediante acuerdo de Consejo de Ministros, el 26 de junio de 1985. desde la estrategia europea medioambiental / Rafael Córdoba Hernández y Agustín Hernández-Aja 
En el año 2006, la red convocó a sus miembros a presentar sus iniciativas. Las actuaciones presentadas tenían que encuadrarse en uno de estos ejes de actuación:

- Transporte: proyectos dirigidos a promover modos de transporte no contaminantes.

- Energía: acciones dirigidas a reducir el consumo de energía final, a la mejora de la eficiencia y a la producción de energía con fuentes renovables.

- Edificación y planificación urbana: actuaciones encaminadas a la reducción de las necesidades energéticas de los edificios y a la incorporación en ellos de energías renovables y acciones dirigidas a reducir las necesidades de desplazamiento y energía de los ciudadanos, manteniendo ciudades compactas, multifuncionales y eficientes.

- Ecotecnología: actuaciones cuyas características tecnológicas permiten una reducción del impacto ambiental de la actividad en cuestión.

Se exhibieron 124 actuaciones de 30 municipios. Ciudades de distintos tamaños como Madrid o Chipiona presentaron sus propuestas al certamen.

OPINIÓN: Revisión de la best practice "red española de ciudades por el clima", desde la estrategia europea medioambiental / Rafael Córdoba Hernández y Agustín Hernández-Aja
En el catálogo finalmente aparecen 40 de ellas: una por municipio y las diez "Buenas prácticas" galardonadas.

Mediante el análisis de la relación de estas prácticas con los objetivos de la estrategia europea, se busca analizar la profundidad y complejidad del desarrollo actual de las políticas municipales a la hora de combatir el cambio climático.

En 2008, la red fue reconocida como una de las diez mejores prácticas mundiales -Best Practices- en el concurso internacional promovido por el Programa de Naciones Unidas para los Asentamientos Humanos (UN-Habitat) ${ }^{5}$.

\section{Análisis de las actuaciones municipales}

De las 40 prácticas incluidas en el catálogo, 5 $(12,5 \%)$ tienen relación con la "Edificación y planificación urbana". En el mismo número se encuentran las relacionadas con la "Ecotecnología" y 10 (25\%) con "Transporte". La mitad restante se refiere a "Energía", centrándose en medidas de reducción del consumo energético y mejora de la eficiencia.

Convocatoria bianual, en la que un jurado independiente elige las mejores prácticas para mejorar las condiciones de vida de la población, ya sea trabajando sobre aspectos como el transporte y la accesibilidad, la vivienda, la gestión de los recursos naturales o la lucha contra la exclusión social, entre otros aspectos.

revista invi № 77 / Mayo 2013 / Volumen N² 28: 229-237 231 
Pueden asociarse las buenas prácticas recogidas en el catálogo a los seis objetivos anteriores. De este análisis se desprende que ninguno de los objetivos de la estrategia europea queda sin abordar, y por tanto, en mayor o menor grado el conjunto de las actuaciones abarca la totalidad de los objetivos del programa.

El 30\% de las actuaciones se centra en reducir las emisiones en el sector energético, el segundo lugar lo ocupan aquellas otras medidas destinadas a lograr el descenso de las emisiones (24\%). Con similar peso (23\%) se encuentran las medidas destinadas a cumplir los compromisos internacionales. Las menos numerosas pasan a ser las iniciativas dirigidas a disminuir las emisiones en transporte (10\%), otros sectores (7\%) y el sector industrial (6\%).

En la tabla siguiente se analiza el grado de homogeneidad de los campos prioritarios de la red con los objetivos de la estrategia europea, contabilizando las prácticas de cada campo asociadas a los seis objetivos europeos, incluyendo el peso porcentual del número de prácticas por objetivo.

Se evidencia cómo las actuaciones destinadas a cumplir los compromisos internacionales (objetivo 1) centran sus esfuerzos en el campo energético (44\%), mientras que los menos lo hacen desde la planificación urbana (12\%). Por su parte, en el objetivo de reducir emisiones en sector energético (objetivo 2) coincide mayoritariamente con las actuaciones del campo del mismo nombre (67\%), aunque en este caso las actuaciones urbanas alcanzan el 19\%. Dentro del objetivo 3. Reducir emisiones en sector transportes, un 93\% de las actuaciones municipales coincide con el área prioritaria del mismo nombre y solo una dentro del área energética. Para lograr el objetivo 4 (reducción de emisiones industriales) se tornan fundamentales las actuaciones asociadas a la ecotecnología. A partes iguales se dividen las actuaciones urbanas y las ecotecnológicas para reducir emisiones en otros sectores (objetivo 5). Dentro del objetivo 6, usar otros instrumentos adecuados, el área mayoritaria es la energética (53\%), seguida de transporte (26\%).

Dada la implicación en los procesos urbanos del transporte y de la planificación urbana, se considera de mayor interés centrarse únicamente en un análisis más profundo de las categorías de edificación y planificación urbana y transporte.

\section{PRÁCTICAS URBANAS EN EDIFICACIÓN Y PLANIFICACIÓN URBANA}

En el catálogo figuran 5 iniciativas, indicando la resistencia existente para crear nuevas estrategias de cambio e innovadoras en un espacio clave para la reducción de emisiones. Esto es más acusado si se tiene en cuenta que solo una actuación está claramente relacionada con la planificación urbana, operando las restantes sobre edificaciones. 


\section{TABLA 1. DISTRIBUCIÓN DE LAS ACTUACIONES SEGÚN CAMPOS PRIORITARIOS Y OBJETIVOS DE LA ESTRATEGIA DE CAMBIO CLIMÁTICO DEL VI PROGRAMA DE ACCIÓN COMUNITARIA, EN MATERIA DE MEDIO AMBIENTE.}

\begin{tabular}{|c|c|c|c|c|c|c|c|c|c|c|c|c|}
\hline & \multicolumn{2}{|c|}{ Objetivo 1} & \multicolumn{2}{|c|}{ Objetivo 2} & \multicolumn{2}{|c|}{ Objetivo 3} & \multicolumn{2}{|c|}{ Objetivo 4} & \multicolumn{2}{|c|}{ Objetivo 5} & \multicolumn{2}{|c|}{ Objetivo 6} \\
\hline & $\mathrm{N}$ & $\%$ & $\mathrm{~N}$ & $\%$ & N & $\%$ & $\mathrm{~N}$ & $\%$ & $\mathrm{~N}$ & $\%$ & $\mathrm{~N}$ & $\%$ \\
\hline $\begin{array}{l}\text { Edificación y planificación } \\
\text { urbana }\end{array}$ & 4 & $12 \%$ & 8 & $19 \%$ & - & - & 1 & $11 \%$ & 5 & $50 \%$ & 2 & $6 \%$ \\
\hline Ecotecnología & 5 & $16 \%$ & 6 & $14 \%$ & - & - & 8 & $89 \%$ & 5 & $50 \%$ & 5 & $15 \%$ \\
\hline Energía & 14 & $44 \%$ & 28 & $67 \%$ & 1 & $7 \%$ & - & - & - & - & 18 & $53 \%$ \\
\hline Transporte & 9 & $28 \%$ & - & - & 14 & $93 \%$ & - & - & - & - & 9 & $26 \%$ \\
\hline Total de actuaciones & 32 & $100 \%$ & 42 & $100 \%$ & 15 & $100 \%$ & 9 & $100 \%$ & 10 & $100 \%$ & 34 & $100 \%$ \\
\hline
\end{tabular}

Fuente: FEMP (2006) Actuaciones urbanas por el Clima. Elaboración propia.

Sólo la premiada está relacionada con el espacio público y el urbanismo. Este proyecto busca darle solución a uno de los problemas que habitualmente se encuentran en el ejercicio de nuestra profesión. El Bosque de los Niños nace como nexo entre dos realidades muy distintas, la del núcleo histórico y la nueva zona del puerto de Sagunto (Valencia). Así, con la creación de un bosque urbano y una zona de transición entre los polígonos industriales y los núcleos de población, consigue integrar ambas realidades. También busca diversificar y mejorar el paisaje urbano y la calidad ambiental municipal, creando un pequeño sumidero de dióxido de carbono.

El resto de las actuaciones presentadas basan su reducción de emisiones en el uso de energías renovables o en el fomento del ahorro energético y de la eficiencia energética.

Ninguna de las prácticas seleccionadas incluye la reducción de las emisiones en el sector transporte (objetivo 3). Aunque se podría entender que este tipo de actuaciones siempre cumplen el requisito de sensibilización ciudadana, parece claro que no todas lo hacen del mismo modo. Aunque a priori se puede pensar que una construcción bioclimática o un parque cumplen con la sensibilización al tratarse de objetos novedosos en la ciudad y que se pueden promocionar como ejemplos, bioclimáticos (edificios) o sumideros (parques), el impacto no supera el tiempo asociado a la publicidad de la inauguración, de manera que solo dos de las prácticas premiadas cumplen ese objetivo. 


\section{PRÁCTICAS URBANAS EN TRANSPORTE}

En la reducción de las emisiones de gases de efecto invernadero, resulta tan importante reducir la dependencia del vehículo privado a motor como promocionar modos de transporte alternativos. Estos sistemas menos contaminantes desde el punto de vista de emisiones de dióxido de carbono abarcan desde la bicicleta hasta el uso del transporte colectivo como el metro o el autobús. En cualquier caso, las medidas para la mejora de la accesibilidad en las ciudades solo serán eficaces si se tienen en cuenta dentro del marco general del sistema de planificación. Las actuaciones pueden clasificarse en tres grupos:

- Involucradas en las restricciones al tráfico. Se encuentran medidas para la creación de áreas de circulación exclusivas para el peatón o la bicicleta. El $40 \%$ de las prácticas presentadas tendrían relación con ello, entre ellas la premiada.

234 revista invi № 77 / Mayo 2013 / Volumen № 28: 229-237
- Referentes al tipo de energía utilizada en el transporte. Entre las distintas iniciativas, se propone para la flota municipal el uso del gas licuado, biodiesel, GNC, hidrógeno o la tracción diesel-eléctrica.

- Otro tipo de medidas como un curso de conducción ecológica, que pretende la reducción de emisiones adoptando una serie de pautas al volante o la implantación del carsharing ${ }^{6}$.

La experiencia galardonada en este apartado fue la presentada por el Ayuntamiento de Vitoria-Gasteiz, el proyecto de Sendas urbanas y servicio municipal de préstamo de bicicletas. Con esta práctica se persigue promocionar el uso de la bicicleta como medio de movilidad que no produce emisiones de dióxido de carbono ni otros gases de efecto invernadero. Con tal fin se pretende dotar a la ciudad de vías de circulación preferente para peatones y ciclistas seguras y articuladas. Los resultados del concurso mostraban que se habían inscrito en el proyecto cerca de 20.000 personas y 50.000 usos

6 El carsharing es un nuevo concepto vinculado a la ecomovilidad. Promueve un uso racional del medio de transporte y ofrece la posibilidad de utilizar el vehículo solo cuando se requiera sin necesidad de ser propietario del mismo. La novedad del servicio frente al contrato que nos pueden dar empresas multinacionales de alquiler de vehículos, radica en la factura del servicio. A través de esta asistencia, la factura no refleja únicamente el uso del vehículo (horas de utilización y recorrido), sino que además manifiesta los costes ocultos del coche (mantenimiento, limpieza, seguro), ayudando a la diferenciación del uso y del abuso del mismo. desde la estrategia europea medioambiental / Rafael Córdoba Hernández y Agustín Hernández-Aja 
en el año 2006, estimándose un aumento cercano al 11\% de la población ciclista en la ciudad.

En ninguna iniciativa se encuentran medidas relativas a la implantación de zonas de convivencia entre el vehículo y el peatón o zonas de templado de tráfico (zonas 30) ${ }^{7}$. Tampoco se habla de medidas disuasorias como los aparcamientos exteriores a las grandes aglomeraciones urbanas o de medidas restrictivas para la circulación de los vehículos en determinadas zonas.

\section{Conclusiones}

Las prácticas presentan un panorama todavía muy limitado de implantación de políticas municipales para frenar el cambio climático, dotando a los ciudadanos de pequeños ejemplos que buscan sensibilizar a la ciudadanía, pero que no suponen aún políticas que reduzcan significativamente las emisiones de gases de efecto invernadero.

Los ciudadanos tienen que afrontar su responsabilidad en las causas del problema, pero necesitan que las administraciones les den el soporte estructural que les permita afrontar el cambio.

7 En relación a la implantación de zonas de tráfico calmado, se puede apreciar una contradicción desde el punto de vista ambiental, ya que la mejora de seguridad vial y calidad de vida derivada de la limitación en la velocidad de circulación puede comportar un aumento de las emisiones de los gases contaminantes, siempre y cuando no se consiga una disminución del número de vehículos motorizados que atraviesan la zona pacificada.
Con el tiempo, la ciudadanía se está concienciando de la importancia de su contribución para evitar el cambio climático, ya que las acciones individuales tienen un importante peso en la reducción de emisiones. Pero resulta evidente que es necesario dar un paso más allá, un salto cualitativo, se ha de exigir a nuestros gobernantes herramientas adecuadas para hacerle frente, promulgar el mensaje de alerta a nuestros vecinos y, principalmente, hacer todo lo que esté en nuestras manos para dejar de emitir cantidades ingentes de gases de efecto invernadero. Por ello es imperioso actuar desde la pequeña escala, y esta es la razón de la relevancia de estas prácticas, ya que permiten aplicar las políticas en la escala más próxima al ciudadano.

El papel de planeamiento urbano en fundamental para evitar este continuo deterioro. El sistema urbano actual está controlado por el sector inmobiliario, que difunde las viviendas y las actividades sobre un territorio colmado de infraestructuras que destruyen su calidad e incentivan la movilidad y el consumo de energía. Este modelo se caracteriza por su necesidad de consumir ingentes cantidades de energía y otros recursos naturales como suelo y materiales. El resultado es una ciudad que

revista invi № 77 / Mayo 2013 / Volumen N 28: 229-237 235 
se difumina en el campo ocupando áreas cada vez más extensas. Esta forma de actuar multiplica el consumo del suelo, de energía y materiales, y por lo tanto de las emisiones de dióxido de carbono a la atmósfera. Los ciudadanos quedan reducidos a la categoría de consumidores, con el correlato de pérdida de calidad de vida. Se incrementa la segregación social al vincularse las clases de menores recursos a las periferias de las grandes ciudades y a los espacios centrales de peor calidad. Todo ello dentro de un notable deterioro del espacio público y de la calidad ambiental por la creciente presencia del automóvil y la reducción de vegetación.

Es necesario reflexionar sobre el modelo de ciudad que hemos construido y sobre la necesidad de reconducirlo a un modelo que se acomode mejor a las necesidades de calidad de vida, cohesión social y protección del medio ambiente, incluyendo la necesaria reducción de emisiones de gases de efecto invernadero. Esta ciudad más sostenible y responsable de sus efectos sobre el medio ambiente se debería caracterizar por tener una compacidad y densidad no reñidas con la complejidad formal, la pluralidad de usos y la heterogeneidad y diversidad. Las emisiones de gases de efecto invernadero serían notablemente inferiores en este modelo, al reducir las necesidades de movilidad y el consumo de energía de las edificaciones. La reducción del tamaño funcional de la mancha urbana reduciría no solo el consumo de suelo (no produciéndose emisiones por cambio de uso del mismo), sino también la energía (la energía requerida para desplazarse crece con la distancia y la velocidad) y los recursos materiales. En este nuevo hábitat sería posible que sus habitantes recuperasen el estatuto de ciudadanía incluyendo la conciencia de la repercusión de sus actividades sobre la totalidad del planeta, asumiendo de esta forma su responsabilidad sobre la estabilización del clima.

Los ayuntamientos poseen medios suficientes para actuar de manera solidaria con las políticas propiciadas desde la UE, tanto por su capacidad de intervenir en los sectores y servicios en los que tienen capacidad de intervención directa (transporte público, residuos, abastecimiento de agua...), como en la determinación de normativas y ordenanzas con capacidad de reducir las emisiones de viviendas e industrias. Pero también con la posibilidad de desarrollar, dentro de sus competencias, una fiscalidad propia que permita reconducir y en su caso compensar los usos y procesos que contienen. Y como herramienta global, la planificación urbana, que tiene tanto la capacidad de reducir los efectos más perniciosos del crecimiento de la ciudad, como de reconvertir el proceso centrándose en la recuperación de la ciudad existente y de los espacios degradados que contiene o la circundan.

Desde la planificación urbana se pueden adoptar planteamientos ecológicos, así como medidas de diseño urbano que permitan las estrategias de la arquitectura bioclimática y la redacción de normas 
que aseguren la climatización natural y la optimización energética de todos los edificios públicos. También se pueden elaborar políticas de planificación basadas en principios a largo plazo con programas estratégicos a corto y retroalimentación continua; elaborar políticas que fomenten la flexibilidad de uso en las zonas y los edificios de la ciudad y promover la edificación ecológica para lograr un diseño de edificios duradero, adaptable y de uso múltiple; evitar en los diferentes planes urbanísticos la adopción de modelos territoriales dispersos que incrementen las necesidades de transporte y la segregación de actividades (trabajo, vivienda, comercio, estudio y ocio); facilitar la creación de pasillos verdes que conecten los diversos espacios verdes de las ciudades con el campo circundante; garantizar que los planes incluyan objetivos tanto nacionales como locales relacionados con la sostenibilidad y deben establecerse indicadores para calibrar la magnitud de los problemas y el grado de éxito obtenido en su resolución o incluir la reducción de emisiones de gases de efecto invernadero entre los objetivos y medidas de todos los planes de ordenación territorial y urbanos, que deberán recoger medidas correctoras.

Desde los planes generales de ordenación urbana se puede definir precisamente el modelo de ciudad que queremos configurar y apostar por el desarrollo vinculado con el medio ambiente, en el cual las emisiones de gases de efecto invernadero no sean incrementadas por la falta de

OPINIÓN: Revisión de la best practice "red española de ciudades por el clima", desde la estrategia europea medioambiental / Rafael Córdoba Hernández y Agustín Hernández-Aja equipamientos o la dispersión en el territorio de nuestros planeamientos.

No se debe olvidar el papel de la Agenda 21 Local. Este es un instrumento de gestión surgido de la Conferencia de Naciones Unidas sobre Medio Ambiente y Desarrollo, que se ha convertido en la herramienta de gestión más empleada por los gobiernos locales para abordar los problemas ambientales, y en el que tiene perfecta cabida la lucha contra el cambio climático.

\section{Bibliografía}

CIFUENTES, María, coord.; CÓRDOBA, Rafael, coord. y GÓMEZ, Gloria, coord. Propuestas para mejorar la calidad de vida en las ciudades. [En línea]. Fundación Alternativas. Diciembre de 2008. ISBN: 978-84-92424-45-0. Disponible en: http://www. falternativas.org/laboratorio/documentos/documentos-de-trabajo/propuestas-para-mejorar-lacalidad-de-vida-en-las-ciudades.

RED Española de Ciudades por el Clima. Actuaciones urbanas por el clima. España, Federación Española de Municipios y Provincias. 2006. 209 p. Biblioteca Ciudades por el Clima No5.

UNIÓN Europea. Decisión No 1600/2002/CE del Parlamento Europeo y del Consejo, de 22 de julio de 2002, por la que se establece el Sexto Programa de Acción Comunitaria en Materia de Medio Ambiente. Diario Oficial de las Comunidades Europeas. L 242, 2001.

revista invi № 77 / Mayo 2013 / Volumen N² 28: 229-237 237 